NATHaLie Leite GazZaneo

Interseções e limites entre a neutralidade da rede e a política de defesa da concorrência no Brasil

\author{
Dissertação de Mestrado \\ Orientador: Professor Dr. Newton Silveira
}

Universidade de São PaUlo

FACULDADE DE DiREITO

SÃo PaUlo/SP

2018 


\section{Interseções e limites entre a neutralidade da rede e a política de defesa da concorrência no Brasil}

Dissertação apresentada a Banca Examinadora do Programa de Pós-Graduação em Direito, da Faculdade de Direito da Universidade de São Paulo, como exigência parcial para a obtenção do título de Mestre em Direito, na área de concentração em Direito Comercial, sob a orientação do Professor Dr. Newton Silveira.

Universidade de São Paulo

FACULdAde de Direito

SÃo PaUlo/SP 


\section{Catalogação da Publicação \\ Serviço de Biblioteca e Documentação \\ Faculdade de Direito da Universidade de São Paulo}

Gazzaneo, Nathalie Leite

Interseções e limites entre a neutralidade da rede e a política de defesa da concorrência no Brasil / Nathalie Leite Gazzaneo ; orientador Newton Silveira -- São Paulo, 2018.

171

Dissertação (Mestrado - Programa de Pós-Graduação em Direito Comercial) Faculdade de Direito, Universidade de São Paulo, 2018.

1. Internet. 2. Neutralidade da rede. 3. Concorrência. I. Silveira, Newton, orient. II. Título. 


\section{DEDICATÓRIA}

Aos meus pais e irmãos, meus maiores incentivadores de sonhos e guias nesta vida, por me ensinarem tudo que eu precisava para ir aonde quer que eu quisesse.

Ao meu grande amor e melhor companheiro de vida, por multiplicar meus motivos para entregar, acreditar, aceitar e agradecer. 


\section{RESUMO}

GAZZANEO, Nathalie L. Interseções e limites entre a neutralidade da rede e a política de defesa da concorrência no Brasil. 2018. 171 f. Dissertação (Mestrado em Direito Comercial) Faculdade de Direito, Universidade de São Paulo, 2018.

O objeto central deste trabalho é expor os principais pontos de interseção entre a neutralidade da rede e a política de defesa da concorrência no Brasil, reconhecendo o valor do diálogo entre estas duas fontes, mas apontando para a existência de limites importantes e individualizantes entre elas. Um dos grandes embates teóricos sobre a necessidade ou não de uma regulação ex ante sobre a neutralidade da rede reside na insuficiência ou suficiência da política de defesa da concorrência para sanar os eventuais efeitos adversos de práticas discriminatórias do tráfego empreendidas por provedores de acesso à Internet. Para além dessa discussão, diversos institutos típicos do direito antitruste estão presentes em análises a respeito da neutralidade da rede, em especial as noções de mercado de múltiplas pontas, de posição dominante, de integrações verticais e práticas discriminatórias e de bem-estar do consumidor. Em suma, as interfaces e possibilidades de confusão entre os dois domínios são inúmeras, sendo necessário estabelecer seus limites mais claramente. De onde vem, o que estabelece e o que busca a regra de neutralidade da rede brasileira? Quais são as suas interfaces com a política de defesa da concorrência no Brasil? Quais são os limites entre a neutralidade da rede e a política de defesa da concorrência no Brasil? Esses são os principais questionamentos enfrentados neste trabalho.

Palavras-chave: Internet. Neutralidade da rede. Concorrência. 


\begin{abstract}
GAZZANEO, Nathalie L. Intersections and limits between net neutrality and competition policy in Brazil. 2018. 171 pages. Master Degree - Law School, University of São Paulo, 2018.

The goal of this study is to present the main points of intersection between net neutrality and competition policy in Brazil, recognizing the value of the dialogue between these two domains, but pointing out to the existence of important and individualizing limits between them. One of the major theoretical clashes about the need for net neutrality ex ante regulation consists in whether or not competition policy can sufficiently address the potential adverse effects of ISPs' traffic discrimination practices. Beyond that, a number of typical antitrust concepts are used to analyze net neutrality, in particular the concepts of multisided markets, dominant position, vertical integration, discriminatory practices and consumer welfare. In short, the interfaces and subsequent risks of confusion between net neutrality and competition policy require a clearer establishment of the limits between them. Where does the Brazilian net neutrality rule come from, what does it impose and what are its goals? What are the interfaces between net neutrality and antitrust policy in Brazil? What are the boundaries between net neutrality and competition policy in Brazil? These are the main questions addressed in this study.
\end{abstract}

Keywords: Internet. Net neutrality. Competition. 


\section{LISTA DE SIGLAS E ABREVIAÇÕES}

Anatel - Agência Nacional de Telecomunicações

BEREC - Body of European Regulators for Electronic Communications

CADE - Conselho Administrativo de Defesa Econômica

CDN - Rede de distribuição de conteúdo

CGI.br - Comitê Gestor da Internet no Brasil

$\mathrm{CP}$ - Provedor de conectividade à Internet; é um tipo de ISP

DIREITO RIO - Escola de Direito do Rio de Janeiro da Fundação Getúlio Vargas

DPDC - Departamento de Proteção e Defesa do Consumidor

ECAD - Escritório Central de Arrecadação e Distribuição (de direitos autorais referentes à execução musical)

EEST - Entidades Exploradores de Serviços de Telecomunicação

FCC - Federal Communications Commission

FTC - Federal Trade Commission

HTML - HyperText Markup Language

IAP - Provedor de acesso à Internet (last mile); é um tipo de ISP

IETF - Internet Engineering Task Force

IP - Internet Protocol

IPTV - Televisão por IP

ISP - Provedor de conexão à Internet; gênero do qual são espécies os IAPs e os CPs

LGT - Lei Geral de Telecomunicações (Lei 9.472, de 16 de julho de 1997)

MCI - Marco Civil da Internet (Lei 12.965, de 23 de abril de 2014)

MCTIC - Ministério da Ciência, Tecnologia, Inovações e Telecomunicações

MPF - Ministério Público Federal

NAT - Network Address Translation

Nic.br - Núcleo de Informação e Coordenação do Ponto BR

ONU - Organização das Nações Unidas 
OECD - Organização para a Cooperação e Desenvolvimento Econômico

OSP - Provedor de serviços online ou Provedor de aplicações

PSCI - Provedores de Serviços de Conexão à Internet

QoS - Qualidade de serviço

RGC - Regulamento Geral de Direitos do Consumidor de Serviços de Telecomunicações

SAL/MJ - Secretaria de Assuntos Legislativos do Ministério da Justiça

SBDC - Sistemas Brasileiro de Defesa da Concorrência

SCI - Serviços de Conexão à Internet

SCM - Serviço de Comunicação Multimídia

SDE - Secretaria de Direito Econômico do Ministério da Justiça

SEAE - Secretaria de Acompanhamento Econômico do Ministério da Fazenda

Senacon - Secretaria Nacional do Consumidor

SG - Superintendência Geral do CADE

SMP - Serviço Móvel Pessoal

STF - Supremo Tribunal Federal

STFC - Serviço Telefônico Fixo Comutado

SVA - Serviço de Valor Adicionado

UE - União Europeia

VoD - Vídeo sob demanda

VoIP - Voz por IP

VoLTE - Voz por evolução em longo prazo

VPN - Virtual private network 


\section{SUMÁRIO}

INTRODUÇÃO

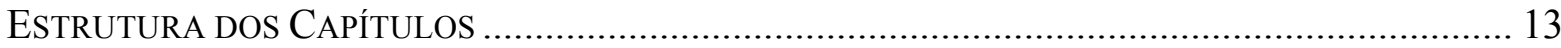

CAPítulo 1 - Histórico, elementos E OBJetivos da Regra de NEUTRALIDAde Da REDE NO BRASIL ....................................................................................................................................... 15

1.1. Neutralidade da Rede e a camada de conteúdo da Internet como espaÇo

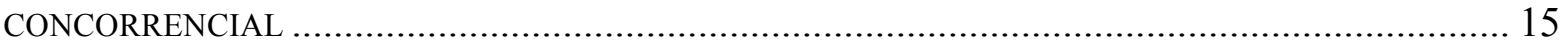

1.2. HistóRICO E ELEMENTOS DA NEUTRALIDADE DA REDE NO BRASIL ……………….......... 23

1.2.1. Protorregulação da neutralidade da rede no setor de telecomunicações ....... 23

1.2.2. O "Decálogo da Internet" do Comitê Gestor da Internet no Brasil ................ 30

1.2.3. Marco Civil da Internet: do anteprojeto de lei ao Decreto 8.771/2016............ 31

1.2.4. Elementos da regra de neutralidade da rede brasileira....................................... 42

1.3. NEUTRALIDADE DA REDE NA EXPERIÊNCIA REGULATÓRIA COMPARADA ............................ 46

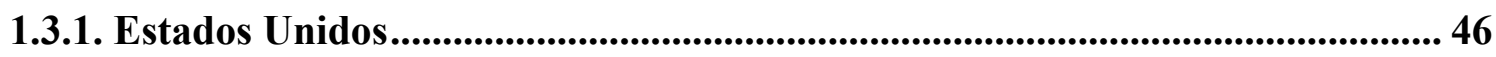

1.3.2. União Europeia ......................................................................................................... 56

1.4. OBJETIVOS DA REGULAÇÃO DA NEUTRALIDADE DA REDE NO BRASIL ................................ 61

1.4.1. Inovação e enfrentamento a barreiras de entrada na camada de conteúdo da

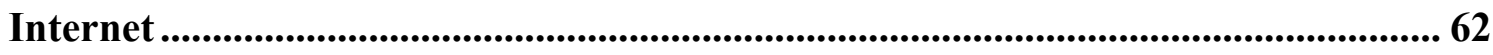

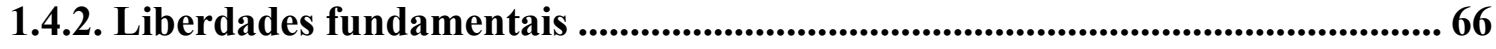

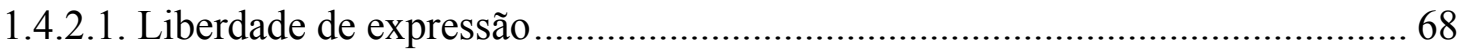

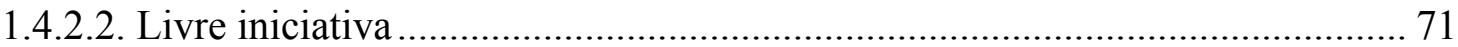

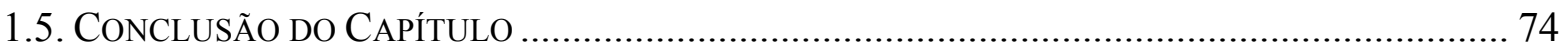

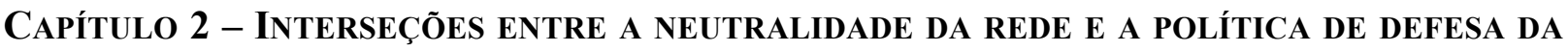
CONCORRENCIA

2.1. QUESTÕES CONCORRENCIAIS NAS DISCUSSÕES SOBRE A REGULAÇÃO DA NEUTRALIDADE DA REDE 75 
2.2. UM PASSO ATRÁS: UM POUCO DE CONTEXTO SOBRE O ECOSSISTEMA DA INTERNET E SEUS

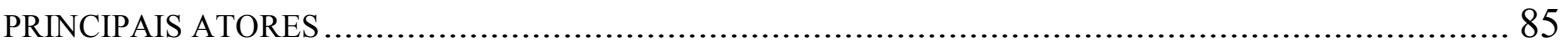

2.2.1. Principais atores do ecossistema da Internet ............................................... 86

2.2.2. Inter-relações entre atores: o ecossistema da Internet como um mercado de múltiplas pontas............................................................................................................... 91

2.3. INSTITUTOS DO ANTITRUSTE COMUNS EM DISCUSSÕES SOBRE NEUTRALIDADE DA REDE... 94 2.3.1. As práticas restritivas da concorrência e os bens jurídicos resguardados pelo direito da concorrência no Brasil................................................................................ 95

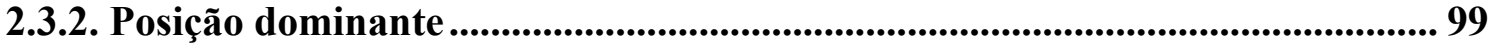

2.3.3. Integrações verticais e práticas discriminatórias ........................................... 103

2.3.3.1. Objetivos das práticas discriminatórias ................................................. 105

2.3.3.1.1. Objetivos determinados em lei como exceções à neutralidade da rede. 105

2.3.3.1.2. Diferenciação de serviços para os consumidores ................................. 106

2.3.3.1.3. Diferenciação de serviços para OSPs ................................................... 107

2.3.3.1.4. Proteção dos negócios ..................................................................... 108

2.3.3.2. Efeitos das práticas discriminatórias para os consumidores ......................... 109

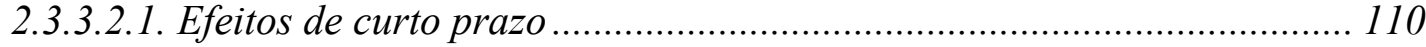

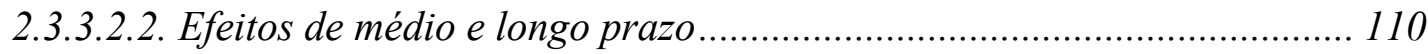

2.3.3.3. Síntese: bjetivos e efeitos das práticas discriminatórias ........................... 111

2.3.4. Addendum: Bem-estar do consumidor........................................................ 112

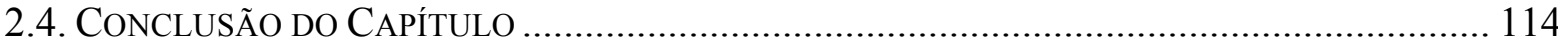

CApítulo 3 - Limites entre a Neutralidade da Rede e a POlítica de defesa da

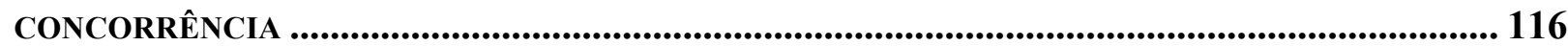

3.1. CONVIVÊnCIA E Limites ENTRE A LEI GERAL DE DEFESA DA CONCORRÊNCIA E LEIS

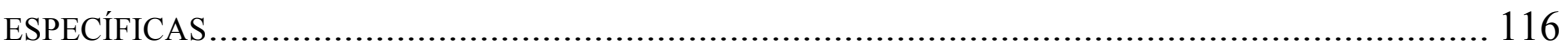

3.1.1. Neutralidade da rede .............................................................................................. 118

3.1.2. Direito do consumidor....................................................................................... 124

3.1.3. Propriedade intelectual .................................................................................... 126

3.2. ColaboraÇão institucional na ATUAÇÃo do Sistema Brasileiro de Defesa da CONCORRENNCIA E O REGIME INSTITUCIONAL COLABORATIVO REFORÇADO PELO DECRETO

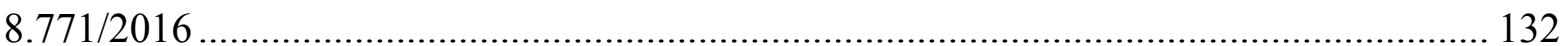


3.3. Limites ENTRE A NEUTRALIDADE DA REDE E A TUTELA CONCORRENCIAL NO BRASIL: O INQUÉRITO ADMINISTRATIVO N 08700.004314/2016-7 ............................................. 137

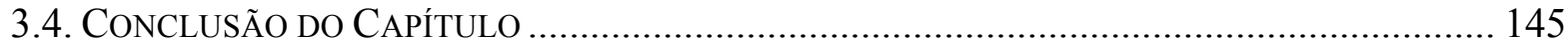

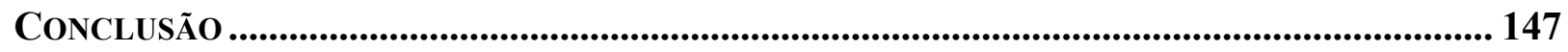

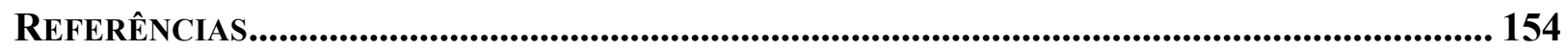




\section{INTRODUÇÃo}

Uma das analogias utilizadas para explicar a arquitetura básica da Internet é a da autoestrada. Os carros em movimento representam o tráfego de dados (necessário a que qualquer click leve o usuário a algum "lugar" na rede) e o ponto de chegada dos carros, para onde eles se movimentam, representa o terminal (celular, computador, tablet, etc.) a que o usuário está conectado. A autoestrada da Internet funciona conforme um manual de instruções chamado "neutralidade da rede", que orienta como o tráfego no trajeto até o ponto de chegada aos usuários finais deve se dar, de modo que todos os carros consigam se mover da forma mais fluida possível, sem que nenhum deles ultrapasse os demais e assim todos alcancem seu ponto de chegada adequadamente.

A neutralidade da rede diz que os operadores que cuidam da autoestrada da Internet não podem bloquear a passagem de determinados carros, nem obrigar determinados carros a andar em menor velocidade, nem oferecer pedágios que permitam que determinados carros circulem por corredores expressos. Há algumas exceções para esses impedimentos, claro. Por exemplo, os carros que estejam empenhados em atender a uma situação de emergência - por exemplo, uma ambulância em operação de socorro - podem ultrapassar os demais. Ainda, o congestionamento do tráfego na autoestrada, impedindo o seu fluxo normal, também autoriza que os operadores da via adotem medidas técnicas pontuais e proporcionais a fim de normalizar a situação. Mesmo nessas exceções, contudo, os operadores da autoestrada devem seguir algumas regras de boa convivência ditadas pelo manual de instruções da neutralidade da rede, como a transparência, por exemplo.

Além da neutralidade da rede, outras regras também se aplicam ao funcionamento da grande autoestrada da Internet. Por exemplo, as regras que tratam da adequada competição entre agentes econômicos, não só nas estradas, mas em qualquer outra forma de atividade econômica. À primeira vista, alguns podem afirmar que o funcionamento da autoestrada dispensaria as orientações da neutralidade da rede, podendo seguir apenas as regras concorrenciais, já que em alguns aspectos elas se assemelham muito com as orientações da neutralidade da rede. Mas, se por um lado as regras concorrenciais são ótimos complementos à neutralidade da rede, ajudando a manter o funcionamento adequado da autoestrada da Internet, 
por outro, os objetivos das regras concorrenciais não coincidem perfeitamente com os da neutralidade da rede, e assim, sozinhas, elas não podem proteger a arquitetura básica da Internet.

Voltando para a perspectiva jurídica, o objeto central deste trabalho é expor os pontos de interseção entre a neutralidade da rede e a política de defesa da concorrência no Brasil, reconhecendo o valor do diálogo entre estas duas fontes, mas apontando para a existência de limites importantes e individualizantes entre elas. Um dos grandes embates teóricos sobre a necessidade ou não de uma regulação ex ante sobre a neutralidade da rede reside justamente na insuficiência ou suficiência da política de defesa da concorrência para sanar os eventuais efeitos adversos de práticas discriminatórias do tráfego pelos provedores de acesso à Internet. Para além dessa discussão, diversos institutos típicos do direito antitruste estão presentes em análises a respeito da neutralidade da rede, em especial as noções de mercado de múltiplas pontas, de posição dominante, de integrações verticais e práticas discriminatórias e de bemestar do consumidor.

Em suma, as interfaces e possibilidades de confusão entre os dois domínios são inúmeras - e tendem a se tornar ainda mais proeminentes em virtude da recente desregulação do núcleo central da regra de neutralidade da rede nos Estados Unidos, cenário no qual ganhará força a atuação da autoridade antitruste daquele país em temas que nos últimos anos estavam limitados à neutralidade da rede. Todos esses elementos contribuem para a relevância e a atualidade do tema das interseções e limites entre a neutralidade da rede e a defesa da concorrência no Brasil.

Ao longo do trabalho, buscamos responder a três ordens de questionamentos: (i) de onde vem, o que estabelece e o que busca a regra de neutralidade da rede brasileira?; (ii) quais são as interfaces entre a neutralidade da rede e a política de defesa da concorrência no Brasil?; e (iii) quais são os limites entre a neutralidade da rede e a política de defesa da concorrência no Brasil?

Metodologicamente, tivemos uma preocupação central em empreender essa análise com bastante atenção na construção da regra de neutralidade da rede brasileira, buscando extrair, tanto quanto possível, conclusões a partir de minutas de regras, relatórios de histórico legislativo, casos e trabalhos que se voltaram para essas discussões no Brasil. A estrutura 
escolhida para a apresentação do trabalho também parte da premissa metodológica de que, no cenário de interseções entre as duas matérias, a melhor forma de encontrar os limites entre a neutralidade da rede e a defesa da concorrência é buscar, primeiro, posicionar muito bem a origem, os elementos e os objetivos da neutralidade da rede no Brasil, para então seguir a exposição sobre suas interfaces com a política de defesa da concorrência e, por fim, propor os limites entre as duas matérias.

Partindo desse exercício, pudemos concluir que os interesses imediatamente protegidos pela norma concorrencial não coincidem perfeitamente com os interesses imediatamente tutelados pela neutralidade da rede. Enquanto a neutralidade da rede busca proteger diretamente a inovação, o enfrentamento a barreiras de entrada na camada de conteúdo da Internet e a liberdade de expressão, incluindo o pluralismo, estes não são interesses diretamente protegidos pela norma concorrencial - embora o direito concorrencial possa, indiretamente, favorecê-los.

Além disso, o direito concorrencial brasileiro comporta outras limitações em relação à neutralidade da rede. No aspecto subjetivo, a Lei 12.529/2011 se dirige diretamente a pessoas físicas ou jurídicas que pratiquem uma atividade econômica em sentido amplo, cuidando diretamente de preocupações de ordem econômica, enquanto a neutralidade da rede, como princípio da disciplina do uso da Internet no Brasil (art. $3^{\circ}$, IV, do Marco Civil da Internet), se direciona imediatamente ao conjunto multissetorial de atores do ecossistema da Internet. Quanto à aplicação das normas, a caracterização do ilícito concorrencial depende necessariamente da delimitação do mercado relevante, ao passo que a neutralidade da rede se aplica ao bloqueio ou degradação discriminatórios do tráfego de quaisquer provedores de aplicações, independentemente da determinação de mercados relevantes.

Também pudemos concluir que, em função desses limites materiais, a execução do princípio neutralidade da rede não se esgota na atuação do Sistema Brasileiro de Defesa da Concorrência, despertando um regime de colaboração institucional entre diferentes entidades, como indica o Decreto 8.771/2016.

\section{Estrutura dos Capítulos}


Desde a promulgação do Marco Civil da Internet (Lei 12.965, de 23 de abril de 2014), o Brasil faz parte de um grupo de algumas dezenas de países ao redor do mundo que regularam expressamente a proteção ao princípio da neutralidade da rede ${ }^{1}$. O Capítulo 1 deste trabalho se dedicará à exposição do histórico da elaboração dessa norma no Brasil - desde a protorregulação do tema no âmbito das telecomunicações até a regulamentação do Marco Civil da Internet por meio do Decreto 8.771/2016. Faremos ainda uma incursão na experiência comparada dos Estados Unidos e da União Europeia, além de introduzir os principais objetivos da regulação da neutralidade da rede, com especial atenção à regra brasileira.

Nos debates sobre a necessidade (ou não) de positivação do princípio da neutralidade da rede, as interfaces com o tema da defesa da concorrência já apareceram com bastante clareza. Não raro, institutos tipicamente concorrenciais - como as noções de mercado de múltiplas pontas, abuso de posição dominante e proteção do bem-estar do consumidor despontaram nesse processo. No caso do Brasil, a própria regra de neutralidade da rede possui considerações concorrenciais (art. $9^{\circ}, \S 2^{\circ}$, IV da Lei 12.965/2016). As interfaces entre esses dois domínios serão objeto do Capítulo 2 desta Dissertação.

Dadas as interfaces entre os dois domínios, é preciso distinguir mais claramente os fins a que cada um deles serve, de modo a garantir a eficácia da sua aplicação. A neutralidade da rede não é o primeiro domínio jurídico cujos limites com a política de defesa da concorrência já precisaram ser mais bem estabelecidos no Brasil - o direito do consumidor e a propriedade intelectual já desafiaram essa necessidade de delimitação e oferecem lições importantes nesse sentido. Além de expor brevemente a experiência brasileira com esses outros domínios, o Capítulo 3 apresentará uma abordagem teórica para a delimitação entre a neutralidade da rede e o direito da concorrência e explorará a experiência recente do CADE no Inquérito Administrativo n. ${ }^{\circ}$ 08700.004314/2016-7, em que o Conselho foi especificamente confrontado com a alegação de infrações à regra de neutralidade da rede e à ordem econômica e teve a oportunidade de esclarecer parâmetros relevantes para a separação entre esses dois domínios.

\footnotetext{
${ }^{1}$ Para acompanhar o mapa dos países que regularam, estão em vias de regular ou não protegem o princípio da neutralidade da rede, $c f$. https://www.thisisnetneutrality.org/. Acesso em: 9 jan. 2018.
} 


\section{CONCluSÃo}

Ao tempo em que o projeto de pesquisa para este trabalho começou a ser elaborado, o Brasil acabava de adotar o Marco Civil da Internet, em meados de 2014, consagrando a regra de neutralidade da rede. No ano seguinte, os Estados Unidos e a Europa também adotariam suas regras e diversos países do mundo voltariam sua atenção para o tema ${ }^{193}$. Foram anos de intensas discussões sobre a neutralidade da rede em diversas partes do mundo, passando pela regulamentação do Marco Civil da Internet no Brasil, pela elaboração das Orientações do BEREC para a implementação da regra de neutralidade da rede europeia em 2016 e, mais recentemente, pela desregulação do tema empreendida pelo FCC nos Estados Unidos, no fim de 2017.

Com a recente mudança, o FCC abandonou o "sistema de crença sobre a inovação"194 na camada de conteúdo da Internet para prestigiar uma visão de que o dever de transparência sobre as práticas de gestão do tráfego, as forças de mercado e a proteção já oferecida pela política de defesa da concorrência seriam capazes de garantir resultados comparáveis aos buscados por meio do núcleo principal da regra de neutralidade da rede. Essa mudança traz renovado interesse para as discussões sobre as interfaces e os limites entre a neutralidade da rede e a política de defesa da concorrência em todo o mundo, em especial, para as discussões sobre as limitações do direito concorrencial na persecução dos objetivos especificamente perseguidos pela neutralidade da rede - mesmo em países como o Brasil, que conta com um dos modelos regulatórios mais sólidos de proteção à neutralidade da rede, fundado em lei federal e decorrente de extenso debate e participação dos diferentes setores da sociedade.

Neste trabalho, investigamos o histórico, os elementos e os objetivos da regra de neutralidade da rede brasileira e, nesse processo, constatamos que diversos institutos típicos do antitruste sempre apareceram nas discussões sobre neutralidade da rede. Igualmente comuns são os estudos que buscaram apontar a suficiência ou não da política de defesa da

\footnotetext{
${ }^{193}$ Para acompanhar o mapa dos países que regularam, estão em vias de regular ou não protegem o princípio da neutralidade da rede, $c f$. https://www.thisisnetneutrality.org/. Acesso em: 9 jan. 2018.

${ }^{194}$ (Wu, 2003, p. 145).
} 
concorrência para alcançar os objetivos regulatórios pretendidos com uma regra de neutralidade da rede própria. Graças a essa sobreposição de institutos concorrenciais nas discussões sobre neutralidade da rede, podemos perceber diversas interseções teóricas entre estes dois domínios. Percebidas essas interfaces, notamos que a aplicação eficaz quer da norma concorrencial quer da neutralidade da rede exige o estabelecimento de limites materiais e institucionais mais claros entre as duas disciplinas.

Em suma, buscamos responder a três ordens de questionamentos: (i) de onde vem, o que estabelece e o que busca a regra de neutralidade da rede brasileira?; (ii) quais são as interfaces entre a neutralidade da rede e a política de defesa da concorrência no Brasil?; e (iii) quais são os limites entre a neutralidade da rede e a política de defesa da concorrência no Brasil? No caminho percorrido para responder a esses questionamentos, chegamos a algumas sínteses conclusivas:

1. A camada de conteúdo da Internet é um espaço natural de inovação e livre concorrência, aberta ao surgimento de novos atores, em que qualquer ator pode desenvolver uma nova aplicação e disponibilizá-la na rede, sem solicitar permissão ou licenças e, graças à neutralidade da rede, sem ter que pagar nada a mais por isso.

2. Não há uma definição universal sobre o princípio da neutralidade da rede, mas em linhas gerais podemos dizer que ele visa a evitar que operadores da infraestrutura da rede discriminem o tráfego de dados necessários a que usuários finais acessem aplicações e conteúdos de sua escolha na Internet, dado que esse tipo de comportamento tende a desencorajar a inovação dos provedores de aplicações e conteúdos, em detrimento de toda a sociedade.

3. Antes da edição do Marco Civil da Internet, o setor de telecomunicações contava com alguns instrumentos esparsos em que era possível observar rudimentos da regulação da neutralidade da rede, além da emergência de alguns casos em que o tema foi discutido de forma incidental. Exemplos dessa protorregulação são a Norma 004/1995 do Ministério das Comunicações, a Lei Geral de Telecomunicações, a Resolução 477/ 2007 (Regulamento do Serviço Móvel Pessoal - SMP) da Anatel, a Resolução 
632/2014 (Regulamento Geral de Direitos do Consumidor de Serviços de Telecomunicações - RGC) da Anatel, a Resolução 614/13 (Regulamento do Serviço de Comunicação Multimídia - SCM) da Anatel e a análise da aquisição da Brasil Telecom pela então Telemar por essa mesma agência. Embora essas regras possam ser vistas como uma origem remota da regulação da neutralidade da rede no país, nenhuma delas chegou a estabelecer o tratamento não discriminatório dos pacotes de dados pelos responsáveis pelo acesso à Internet na última milha de forma ampla e com implicações multissetoriais.

4. A neutralidade da rede brasileira, resultante do Marco Civil da Internet e regulamentada pelo Decreto 8.771/2016, é antes de tudo um princípio do uso da Internet no Brasil (art. $3^{\circ}$ do Marco Civil) e se direciona diretamente ao conjunto multissetorial de atores do ecossistema da Internet. Na sua concreção, o art. $9^{\circ}$ do Marco Civil se direciona aos provedores de acesso à Internet, isto é, aqueles que fazem a Internet chegar aos usuários finais, na última milha. Seus principais elementos incluem: (i) o mandamento geral de não-discriminação dos pacotes de dados na Internet; (ii) a vedação ao bloqueio, monitoramento, filtragem ou análise do conteúdo dos pacotes de dados; (iii) a previsão de exceções ao mandamento geral de nãodiscriminação ou degradação; e (iv) a previsão de requisitos de validade para o exercício dessas exceções. Tais requisitos de validade incluem (iv.1) a abstenção de causar danos aos usuários; (iv.2) o dever de agir com proporcionalidade, transparência e isonomia e (iv.3) o dever de agir em condições comerciais não discriminatórias, "abstendo-se de praticar condutas anticoncorrenciais" (art. $9^{\circ}, \S 2^{\circ}$ do Marco Civil). Este último requisito de validade já revela a primeira interface importante entre a regra de neutralidade da rede brasileira e a política de defesa da concorrência.

5. O estudo do histórico legislativo do Marco Civil da Internet, em especial do parecer final ao PL 2.126/2011 (que deu origem à lei), permite concluir que os principais objetivos diretamente perseguidos pela regra de neutralidade da rede brasileira são: (i) a inovação e o enfrentamento a barreiras de entrada na camada de conteúdo da Internet; (ii) a liberdade de expressão; e (iii) a livre inciativa. 
6. Um dos grandes embates teóricos sobre a necessidade ou não de uma regulação ex ante sobre a neutralidade da rede reside na insuficiência ou suficiência da política de defesa da concorrência para sanar os eventuais efeitos adversos de práticas discriminatórias do tráfego pelos provedores de acesso à Internet - eis a terceira interseção que identificamos entre os dois temas. No Brasil, contudo, o próprio Sistema Brasileiro de Defesa da Concorrência, por meio da SEAE, concluiu e opinou publicamente no sentido de que a existência de uma regra de neutralidade da rede era oportuna e benéfica para o país.

7. Para além da discussão sobre a suficiência ou não da política de defesa da concorrência para alcançar os objetivos perseguidos diretamente pela neutralidade da rede, a quarta interface entre estes dois domínios é representada pelos diversos institutos típicos do direito antitruste presentes em análises a respeito da neutralidade da rede, em especial as noções de mercado de múltiplas pontas, de posição dominante, de integrações verticais e práticas discriminatórias e de bem-estar do consumidor.

8. A análise das inter-relações entre os principais atores do ecossistema da Internet (ISPs, OSPs e usuários finais), de fato, revela que esse ecossistema pode ser visto como um mercado de múltiplas pontas, com externalidades diretas e indiretas entre seus atores.

9. Olhando para o contexto brasileiro, os dados da Anatel sobre os mercados de banda larga fixa e de telefonia móvel, incluindo as tecnologias de acesso à Internet móvel, apontam para um cenário de grande concentração, em que as três empresas com maior fatia em cada um desses mercados detêm posição dominante. Do ponto de vista da neutralidade da rede, a posição dominante detida por provedores de acesso à Internet é uma preocupação apriorística, porque gera um incentivo a que eles se comportem como gatekeepers, isto é, discriminando de forma irregular o tráfego de dados de determinadas aplicações e conteúdos que chegariam aos usuários finais por meio de suas redes. Já na perspectiva concorrencial, a questão central reside em identificar, ex post, se tais provedores de acesso efetivamente abusam ou possuem incentivos suficientes para abusar da sua posição dominante, levando em consideração que eles operam em um mercado de múltiplas pontas. 
10. A detenção de posição dominante, aliada a cenários de integração vertical, gera diferentes incentivos à prática de condutas discriminatórias. Integrações verticais per se não são uma violação apriorística quer à neutralidade da rede quer ao direito concorrencial. Com base na regra de neutralidade da rede brasileira, para que essas integrações sejam um ilícito é preciso que tenham por objeto a priorização de pacotes de dados específicos decorrentes da verticalização (Decreto 8.771/2016, art. $9^{\circ}$, II e III). Já para o direito concorrencial, a discriminação resultante de uma verticalização dessa natureza não necessariamente consistiria em uma infração à ordem econômica a análise concorrencial dessas práticas dependeria da observação de fatores adicionais, como a posição de mercado do respectivo provedor de acesso e os efeitos dessas práticas para os mercados relevantes e para os consumidores afetados.

11. Por fim, o tema do bem-estar do consumidor é mais um ponto de interface entre a neutralidade da rede e o direito da concorrência. Nem a neutralidade da rede nem o direito da concorrência protegem os interesses dos consumidores diretamente, mas ambos tendem a favorecer a ampliação do seu poder de escolha. No caso da neutralidade da rede, esse efeito decorre da preservação da qualidade e da diversidade dos produtos e serviços disponibilizados aos consumidores na camada de conteúdo da Internet e, dependendo da conformação das práticas diferenciais executadas pelos ISPs, do controle dos preços praticados para o acesso à Internet pública.

12. Diante dessas diversas interfaces, a aplicação eficaz quer da norma concorrencial quer da neutralidade da rede exige o estabelecimento de limites materiais e institucionais mais claros entre as duas disciplinas. $\mathrm{O}$ estabelecimento de limites materiais entre o direito concorrencial e outras leis específicas - como o direito do consumidor e a propriedade intelectual, por exemplo - depende de um exercício de identificação de objetivos mediatos e imediatos de cada uma dessas matérias, decorrentes, na lição de Carnelutti, dos diferentes interesses mediata ou imediatamente protegidos por cada disciplina jurídica.

13. Partindo desse exercício, podemos concluir que os interesses imediatamente protegidos pela norma concorrencial não coincidem perfeitamente com os interesses 
imediatamente tutelados pela neutralidade da rede. Enquanto a neutralidade da rede busca proteger diretamente a inovação, o enfrentamento a barreiras de entrada na camada de conteúdo de forma apriorística e a liberdade de expressão, incluindo o pluralismo, estes não são interesses diretamente protegidos pela norma concorrencial. A livre iniciativa seria o único interesse diretamente protegido por ambos os domínios jurídicos no Brasil, mas uma análise mais cautelosa mostra que enquanto o sentido da proteção à livre concorrência pela neutralidade da rede se concentra na liberdade dos modelos de negócios promovidos na Internet, o direito concorrencial busca proteger a livre iniciativa de forma ampla, no sentido de assegurar a todos a faculdade de explorar qualquer atividade econômica com finalidade de lucro, sem favorecimento apriorístico de um setor econômico em detrimento de outro. A inovação, a seu turno, embora seja diretamente protegida pela neutralidade da rede, é protegida apenas indiretamente pelo direito concorrencial brasileiro. E a proteção aos consumidores, embora seja um ponto de interface entre estes dois conjuntos de normas, não constitui um interesse imediatamente protegido por nenhum deles. Assim, a análise de objetivos diretos perseguidos por cada norma permite concluir que no Brasil, o direito da concorrência é insuficiente para proteger, sozinho, os objetivos diretamente perseguidos pela neutralidade da rede.

14. Além disso, o direito concorrencial brasileiro comporta outras limitações em relação à neutralidade da rede. No aspecto subjetivo, a Lei 12.529/2011 se dirige diretamente a pessoas físicas ou jurídicas que pratiquem uma atividade econômica em sentido amplo, cuidando diretamente de preocupações de ordem econômica. Já a neutralidade da rede, como princípio da disciplina do uso da Internet no Brasil (art. $3^{\circ}$, IV, do Marco Civil da Internet), se direciona imediatamente ao conjunto multissetorial de atores do ecossistema da Internet. Quanto à aplicação das normas, a caracterização do ilícito concorrencial depende necessariamente da delimitação do mercado relevante, ao passo que a neutralidade da rede se aplica ao bloqueio ou degradação discriminatórios do tráfego de quaisquer provedores de aplicações, independentemente da determinação de mercados relevantes. Em função desses limites, a execução do princípio neutralidade da rede não se esgota na atuação do SBDC, despertando um regime de colaboração institucional entre diferentes entidades, como indica o Decreto 8.771/2016. 
15. A experiência recente do CADE em caso envolvendo a análise de ofertas comerciais com interfaces com a neutralidade da rede (Inquérito Administrativo n. ${ }^{\circ}$ 08700.004314/2016-7) ajudou a ilustrar não apenas os limites materiais entre a neutralidade da rede e a política de defesa da concorrência no Brasil, como também os limites da atuação institucional da autoridade antitruste nesses casos, prestigiando o modelo colaborativo previsto no Decreto 8.771/2016. 


\section{REFERÊNCIAS}

AlmEIDA, Guilherme. Marco Civil da Internet: Antecedentes, Formulação Colaborativa e Resultados Alcançados. In: ARTESE, Gustavo (Org.). Marco Civil da Internet: análise jurídica sob uma perspectiva empresarial. São Paulo: Quartier Latin, 2015, pp. 19-63.

Anatel, Anatel esclarece uso de VoIP para oferta de serviço de voz, 2005. Disponível em: http://bit.ly/29Hshq7. Acesso em: 9 jan. 2018.

. Resolução 477, 2007. Disponível em: http://bit.ly/29N0qX6 . Acesso em: 9 jan. 2018.

. Resolução 632, 2014. Disponível em: http://bit.ly/2aeaYfU . Acesso em: 9 jan. 2018.

. Ato n. 7.828, 2008. Disponível em: http://bit.ly/29OaWwI. Acesso em: 9 jan. 2018.

. Voto da Conselheira Maria Emília Curi no Procedimento de Anuência Prévia que analisou as operações societárias para aquisição, por parte da TELEMAR, de ações representativas do controle de emissão da INVITEL, implicando a aquisição do controle indireto do Grupo BRT pelo Grupo TELEMAR. Disponível em: http://bit.ly/2a22uMQ. Acesso em: 9 jan. 2018.

2018.

. Consulta Pública n. 45, 2011. Disponível em: http://bit.ly/2a0FqeG. Acesso em: 9 jan.

. Resolução 614, 2013. Disponível em: http://bit.ly/1KB3927. Acesso em: 9 jan. 2018.

.Dados - Banda larga - Acessos, 4 out. 2017. Disponível em: http://bit.ly/2yJbP3Z. Acesso em: 9 jan. 2018.

. Dados - Telefonia móvel - Acessos, 8 nov. 2017. Disponível em: http://bit.ly/2gwzyyr. Acesso em: 9 jan. 2018.

2018.

. Resolução no 574, 2011. Disponível em: http://bit.ly/2B8DUTA. Acesso em: 9 jan. 
2018.

Resolução no 575, 2011. Disponível em: http://bit.ly/2zZn89G. Acesso em: 9 jan.

. Dados - Qualidade dos Serviços, 30 nov. 2017. Disponível em: http://bit.ly/2zTmDQl. Acesso em: 9 jan. 2018.

2018.

Resolução no 581, 2012. Disponível em: http://bit.ly/2mLkwsV. Acesso em: 9 jan. . Resolução 410, 2005. Disponível em: http://bit.ly/2E8vkVZ. Acesso em: 9 jan. 2018.

Análise $\mathrm{n}^{\circ}$ 100/2016/SEI/AD, 9 nov. 2016. Disponível em: http://bit.ly/2EjcxY5. Acesso em: 9 jan. 2018.

. Informe $n^{\circ}$ 4/2016/SEI/SCP, 6 set. 2016. Disponível em: http://bit.ly/2EjcxY5. Acesso em: 9 jan. 2018.

Aranha, Marcio Iorio. Manual de Direito Regulatório, $3^{\text {a }}$ ed. Laccademia Publishing: Londres, 2015a.

Direito das Telecomunicações, $3^{a}$ ed. Londres: Laccademia Publishing, $2015 b$.

AsCarelli, Tullio. Problemas das Sociedades Anônimas e Direito Comparado. São Paulo: Saraiva, 1969.

Teoria Della Concorrenza e dei Beni Immaterialli, $3^{\text {a }}$ ed. Milão: A. Giuffrè, 1960.

BARZILAI-NAHON, Karine. Toward a theory of network gatekeeping: a framework for exploring information control. Journal of the American Society for Information Science and Technology, Hoboken, v. 59(9), 2008, pp. 1493-1512.

BeChaRA, Marcelo; Borges, Luana. O Marco Civil da Internet e o setor de telecomunicações. In: Artese, Gustavo. Marco Civil da Internet - análise jurídica sob uma perspectiva empresarial. São Paulo: Quartier Latin, 2015, pp. 359-379. 
Belli, Luca. Network Neutrality and Human Rights An Input Paper. In: Belli, Luca; FILIPPI, Primavera De (Eds.). The value of Network Neutrality for the Internet of Tomorrow: Report of the Dynamic Coalition on Network Neutrality. [S.1]: HAL Archives Ouvertes, 2014, pp. 11-18. Disponível em: http://bit.ly/2zySsyo. Acesso em: 9 jan. 2018.

; MARSDEn, Christopher. Not Neutrality but 'Open Internet' à 1'Européenne, 2015. Disponível em: http://bit.ly/2cYQTP1 . Acesso em: 9 jan. 2018.

BENKLER, Yochai. The Wealth of Networks: How Social Production Transforms Markets and Freedom. New Haven: Yale University Press, 2006. Disponível em: http://bit.ly/1eKEJz5. Acesso em: 9 jan. 2018.

. From consumers to users: shifting the deeper structures of regulation toward sustainable commons and user access. Federal Communication Law Journal, Washington D.C., v. 52, 2000, pp. 561-579.

BEREC. Guidelines on the implementation by National Regulators of European NN rules, 2016. Disponível em: http://bit.ly/2bOVMIZ. Acesso em: 9 jan. 2018.

. Report on differentiation practices and related competition issues in the scope of net neutrality, 2012. Disponível em: http://bit.ly/2AgNG9o. Acesso em: 9 jan. 2018.

BRADEN, Robert. Requirements for Internet Hosts - Communication Layers, 1989. Disponível em: http://bit.ly/2A5jvxw. Acesso em: 9 jan. 2018.

BRANDOM, Russell. Netflix is paying off Comcast for direct traffic access. The Verge, 23 fev. 2014. Disponível em: http://bit.ly/1bBc971. Acesso em: 9 jan. 2018.

Breland, Ali. Net neutrality comments top 20 million. The Hill, 17 ago. 2017. Disponível em: http://bit.ly/2ij3LTK. Acesso em: 9 jan. 2018.

BRIGGS, Asa; BURKE, Peter. Uma história social da mídia - de Gutenberg à Internet, Trad. Maria Carmelita Pádua Dias, $2^{\mathrm{a}}$ ed. Rio de Janeiro: Zahar, 2006.

CADE. Voto do Conselheiro Relator Márcio Oliveira Júnior no Processo Administrativo ${ }^{\circ}$ 08012.003918/2005-04 (Caso Telemar/Vésper), 11 mar. 2015. Disponível em: http://bit.ly/2yI1FQX. Acesso em: 9 jan. 2018. 
. Voto da Conselheira Ana Frazão no Processo Administrativo n ${ }^{\circ}$ 08012.003918/200504 (Caso Telemar/Vésper), 11 mar. 2015. Disponível em: http://bit.ly/2hCiwBx. Acesso em: 9 jan. 2018.

. Processo Administrativo $\mathrm{n}^{\circ}$ 08012.003745/2010-83 (Caso ECAD). Disponível em: http://bit.ly/2jabuRs. Acesso em: 9 jan. 2018.

. Voto do Conselheiro Elvino de Carvalho Mendonça no Processo Administrativo $\mathrm{n}^{\circ}$ 08012.003745/2010-83 (Caso ECAD), 6 mar. 2013. Disponível em: http://bit.ly/2AFdSqB. Acesso em: 9 jan. 2018.

Ato de Concentração $n^{\circ}$ 08012.003107/2010-62 (Caso Oi/Phorm). Disponível em: http://bit.ly/2yXHbTx. Acesso em: 9 jan. 2018.

- Voto do Conselheiro Magalhães Furlan no Ato de Concentração $n^{\circ}$ 08012.003107/2010-62 (Caso Oi/Phorm), 6 out. 2010. Disponível em: http://bit.ly/2ByLMyI. Acesso em: 9 jan. 2018.

. Ato de Concentração no 08012.010585/2010-29 (Caso Telefônica/Phorm). Disponível em: http://bit.ly/2kkVPjq. Acesso em: 9 jan. 2018.

- Voto do Conselheiro Ricardo Machado Ruiz no Ato de Concentração $\mathrm{n}^{\mathrm{o}}$ 08012.010585/2010-29 (Caso Telefônica/Phorm), 27 jul. 2011. Disponível em: http://bit.ly/2kMS6KD. Acesso em: 9 jan. 2018.

. Nota Técnica $\mathrm{n}^{\circ}$ 34/2017/CGAA4/SGA1/SG/CADE no Inquérito Administrativo $\mathrm{n}^{\circ}$ 08700.004314/2016-7, 31 ago. 2017. Disponível em: http://bit.ly/2mcIOcr. Acesso em: 9 jan. 2018.

. Nota Técnica ${ }^{\circ}$ 12/2016/CGAA4/SGA1/SG/CADE no Inquérito Administrativo ${ }^{\circ}$ 08012.011881/2007-41, 24 mai. 2016. Disponível em: http://bit.ly/2CP6ld4 . Acesso em: 9 jan. 2018.

. Resolução no 20, de 9 de junho de 1999. Disponível em: http://bit.ly/2qIuEEQ. Acesso em: 9 jan. 2018. 
CÂMARA DOs Deputados, Substitutivo oferecido em Plenário em substituição à Comissão Especial destinada a proferir Parecer ao Projeto de Lei n. 2.126, de 2011 do Poder Executivo, 2014. Disponível em: http://bit.ly/2ct00Z2. Acesso em: 9 jan. 2018.

CARnElutTI, Francesco. Sistema di diritto processuale civile, v. 1. Pádua: CEDAM, 1936.

Carvalho, Vinicius Marques de. Princípios e finalidade da defesa da concorrência. In: et al, Defesa da concorrência: estudos e votos. São Paulo: Singular, 2015a, pp. 1743.

O sistema brasileiro de defesa da concorrência. In: COELHO, Fabio Ulhoa (Coord.). Tratado de Direito Comercial, v. 6, 2015b, pp. 363-388.

. O que buscamos proteger com a neutralidade de rede. Valor Econômico, 3 jan. 2018. Disponível em: http://bit.ly/2m3Lw3m. Acesso em: 9 jan. 2018.

CB INSIGHTS. The global unicorn club - current private companies valued at $\$ 1 \mathrm{~B}+$. Disponível em: http://bit.ly/1Hctwdu. Acesso em: 9 jan. 2018.

CERQueIRA DE SouzA, Adriana; Nunes Junior, Vidal Serrano. Livre inciativa, livre concorrência e a defesa do consumidor como fundamentos do Marco Civil. In: In: LEMOS, Ronaldo; LeITE, George Salomão (Coord.). Marco Civil da Internet. São Paulo: Atlas, 2014, pp. 105-109.

CGI.br. Resolução CGI.br/RES/2009/003/P, 2009. Disponível em: http://bit.ly/29zap3r. Acesso em: 9 jan. 2018.

Resolução CGI.br/RES/2012/008/P, 2012. Disponível em: http://bit.ly/2D6yZUp. Acesso em: 9 jan. 2018. jan. 2018.

CGI.br/RES/2012/008/P, 2012. Disponível em: http://bit.ly/2D6yZUp. Acesso em: 9

CHOI, Jay Pil; KIM, Byung-Cheol. Net Neutrality and Investment Incentives, 2010. Disponível em: http://bit.ly/2CMvseb. Acesso em: 9 jan. 2018. 
Cintra, Maria Eduarda. Neutralidade de Rede: o caso Comcast v. Netflix e o Marco Civil da Internet. Revista de Direito, Estado e Telecomunicações, Brasília, v. 7, n. 1, 2015, pp. 145170.

Colon, Leandro. Governo combaterá pressão contra a isonomia na internet, diz Kassab. Folha de São Paulo, 5 jan. 2018. Disponível em: http://bit.ly/2CLEKdL. Acesso em: 9 jan. 2018.

Comparato, Fábio Konder. A proteção do consumidor. Importante capítulo do direito econômico. In: MARQues, Claudia Lima; MirageM, Bruno (Orgs.). Direito do Consumidor: fundamentos do direito consumidor. São Paulo: Revista dos Tribunais, 2011.

CONWAY, Carol Elizabeth. Livre concorrência e Internet: a importância da neutralidade de redes para o contínuo desenvolvimento da Web. In: LuCCA, Newton de et al. Direito e Internet III - Tomo II. São Paulo: Quartier Latin, 2015, pp. 88-108.

CRUZ, Francisco Carvalho de Brito. Direito, Democracia e Cultura Digital - a experiência de elaboração legislativa do Marco Civil da Internet. 2013. 138 f. Dissertação (Mestrado em Direito) - Faculdade de Direito, Universidade de São Paulo, São Paulo, 2015.

DPDC. Nota Técnica n 29/2011- CGSC/DPDC/MJ (Caso Telefônica/Phorm), 25 abr. 2011. Disponível em: http://bit.ly/2kMS6KD. Acesso em: 9 jan. 2018.

Nota Técnica $n^{\circ}$ 137/2014-CGCTPA/DPDC/Senacon/MJ (Caso Oi/Phorm), 22.07.2014. Disponível em: http://bit.ly/2BLNvDi. Acesso em: 9 jan. 2018.

ECONOMIDES, Nicholas; TAG, Joacim. Network neutrality on the Internet: a two-sided Market analysis. Information Economics and Policy, Amsterdam, v. 24, pp. 91-104, 2012.

; Hermalin, Benjamin. The economics of network neutrality. RAND Journal of Economics, Santa Monica, v. 43, 2012, pp. 602-629.

ENOMOTO, Lívia Yuri de Queiroz. Influência e disputa regulatória: a atuação de grupos de interesses do setor privado na definição da neutralidade de rede no Brasil. $2017.87 \mathrm{f}$. Dissertação (Mestrado em Ciência Política) - Faculdade de Filosofia, Letras e Ciências Humanas, Universidade de São Paulo, São Paulo. 2017.

Estados Unidos, Tribunal de Apelação do Distrito de Columbia. Comcast v. FCC, 2010. 
Disponível em: http://bit.ly/2cvcQkp. Acesso em: 9 jan. 2018.

, Tribunal de Apelação do Distrito de Columbia. Verizon v. FCC, 2014. Disponível em: http://bit.ly/1ZYEj1m. Acesso em: 9 jan. 2018.

, Suprema Corte, N. Pac. Ry. Co. v. United States, 1957.

, Suprema Corte, White Motor Co. v. United States, 1963.

Evans, David. Multisided platforms, dynamic competition and the assessment of market power for Internet-based firms. In: Aitor Ortiz (Ed.). Internet, competition and regulation of online platforms. Boston: CPI, 2016, pp. 78-94. Disponível em: http://bit.ly/2pIx9p7. Acesso em: 9 jan. 2018.

; SCHMAlensee, Richard. Matchmakers - the new economics of multisided platforms. Boston: Harvard Business Review Press, 2016.

FCC. Madison River Communications, LLC and affiliated companies' Consent Decree, 2005. Disponível em: http://bit.ly/2cpVsQC. Acesso em: 9 jan. 2018.

Commission orders Comcast to end discriminatory network management practices, 2008. Disponível em: http://bit.ly/2cpWlsb. Acesso em: 9 jan. 2018.

. Verizon Wireless to pay $\$ 1.25$ million to settle investigation into blocking of consumers' access to certain mobile broadband applications, 2012. Disponível em: http://bit.ly/2cYh48o. Acesso em: 9 jan. 2018.

. Protecting and Promoting the Open Internet Order, 2015. Disponível em: http://bit.ly/1NOC8bv. Acesso em: 9 jan. 2018.

Appropriate Framework for Broadband Access to the Internet over Wireline Facilities ("Internet Policy Statement"), 2005. Disponível em: http://bit.ly/1UTEVo1. Acesso em: 9 jan. 2018.

2018.

Open Internet Order, 2010. Disponível em: http://bit.ly/1C35jEq. Acesso em: 9 jan. 
. Protecting and Promoting the Open Internet Order, 2015. Disponível em: http://bit.ly/1NOC8bv. Acesso em: 9 jan. 2018.

. Statement of Ajit Pai Commissioner, Federal Communications Commission hearing before the Subcommittee on Communications and Technology of the United States House of Representatives Committee on Energy and Commerce ("Oversight of the Federal Communications Commission"), 2012. Disponível em: http://bit.ly/2iDYqn4. Acesso em: 9 jan. 2018.

. Remarks of FCC Chairman Ajit Pai at the Newseum The Future of Internet Freedom, 2017. Disponível em: http://bit.ly/2gTJpQZ. Acesso em: 9 jan. 2018.

. Notice of Proposed Rulemaking - Restoring Internet Freedom, 2017. Disponível em: http://bit.ly/2z1mEla. Acesso em: 9 jan. 2018.

. Fact Sheet - Restoring Internet Freedom Order, 2017. Disponível em: http://bit.ly/2jhLQdo. Acesso em: 9 jan. 2018.

. Press release - FCC acts to restore internet freedom - Reverses Title II Framework, Increases Transparency to Protect Consumers, Spur Investment, Innovation, and Competition, 2017. Disponível em: http://bit.ly/2ktXK4d . Acesso em: 9 jan. 2018.

. Restoring Internet Freedom Order, 2017. Disponível em: http://bit.ly/2F6DvmY. Acesso em: 9 jan. 2018.

Restoring Internet Freedom FCC-FTC Memorandum of Understanding, 2017. Disponível em: http://bit.ly/2F68BLq. Acesso em: 9 jan. 2018.

FARACO, Alexandre Ditzel. Regulação e direito concorrencial (as telecomunicações). São Paulo: Livraria Paulista, 2003.

FAUTH, Luiz Fernando. Neutralidade da rede e liberdade de expressão: uma abordagem a partir da análise econômica do direito. 2012. 161 f. Dissertação (Mestrado em Direito) Instituto Brasiliense de Direito Público, Brasília, 2012.

Ferraz Junior, Tercio Sampaio. A economia e o controle do Estado. O Estado de São Paulo, 4 jun.1989. 
FERronato, Fernanda. Regulação e Poder na Internet: o Direito Antitruste como alternativa à imposição da Neutralidade da Rede proposta pelo Marco Civil, 2014. Disponível em: http://bit.ly/2EMs6sb. Acesso em: 9 jan. 2018.

Folma Online, Brasil Telecom bloqueia soft de telefonia via web, dizem usuários, 3 nov. 2004. Disponível em: http://bit.ly/29C7Hpv. Acesso em: 9 jan. 2018.

Forgioni, Paula. Os fundamentos do antitruste, $8^{\mathrm{a}}$ ed. São Paulo: Revista dos Tribunais, 2015.

; MIURA, Maira Yuriko Rocha. O princípio da neutralidade e o Marco Civil da Internet no Brasil. LuCCA, Newton de et al. Direito e Internet III - Tomo II. São Paulo: Quartier Latin, 2015, pp. 109-136.

FrAZÃO, Ana. The relation between antitrust and intellectual property on CADE's case law. In: SilveirA, Paulo Burnier. Competition Law and Policy in Latin America: recent developments. Alphen aan den Rijn: Kluwer, 2017, pp. 255-267. Disponível em: http://bit.ly/2F3Eu7g. Acesso em: 9 jan. 2018.

FunG, Brian. The FCC just voted to repeal its net neutrality rules, in a sweeping act of deregulation. The Washington Post, $14 \mathrm{dez}$. 2017. Disponível em: http://wapo.st/2EErDIn. Acesso em: 9 jan. 2018.

Gerlach, Jan. The Informational Ecosystem of Net Neutrality - A Comparison of Regulatory Discourses in the U.S. and the E.U. 2016. 334 f. Tese (Doutorado em Direito), School of Management, Economics, Law, Social Sciences and International Affairs, University of St. Gallen, St. Gallen, 2016.

GetschKo, Demi. As origens do Marco Civil da Internet. In: Lemos, Ronaldo; Leite, George Salomão (Coord.). Marco Civil da Internet. São Paulo: Atlas, 2014, pp. 12-17.

In: Artese, Gustavo. Marco Civil da Internet - análise jurídica sob uma perspectiva empresarial. São Paulo: Quartier Latin, 2015a, pp. 65-71.

. O Marco Civil não é a cura de todos os males. Estadão, 9 fev. 2015b. Disponível em: http://bit.ly/1zOvJ8P. Acesso em: 9 jan. 2018. 
. Neutralidade da rede está segura no Brasil. Estadão, 18 dez. 2017. Disponível em: http://bit.ly/2pIRA6d. Acesso em: 9 jan. 2018.

GILROY, Angele. Access to broadband networks: the net neutrality debate, 2011. Disponível em: http://bit.ly/2CK2xqk. Acesso em: 9 jan. 2018.

GRAU, Eros Roberto. A ordem econômica na Constituição de 1988, $17^{\mathrm{a}}$ ed. São Paulo: Malheiros, 2015.

; Forgioni, Paula. O Estado, a empresa e o contrato. São Paulo: Malheiros, 2005.

GraU-KunTZ, Karin. O desenho industrial como instrumento de controle econômico no mercado secundário de peças de reposição de automóveis - uma análise crítica à recente decisão da Secretaria de Direito Econômico (SDE). Revista de Direito Mercantil, Industrial, Econômico e Financeiro, São Paulo, v. 145, 2007, pp. 148-184.

Heather, Greenfield. CCIA Welcomes Net Neutrality Guidelines from EU Telecom Regulators, 06 set. 2016. Disponível em: http://bit.ly/2cWX8jv. Acesso em: 9 jan. 2018.

KANG, Cecilia F.C.C. Plans Net Neutrality Repeal in a Victory for Telecoms. The New York Times, 21 nov. 2017. Disponível em: http://nyti.ms/2zwgo5U. Acesso em: 9 jan. 2018.

KASTRENAKES, Jacob. ISPs won't promise to treat all traffic equally after net neutrality. The Verge, 15 dez. 2017. Disponível em: http://bit.ly/2AUDkMT. Acesso em: 9 jan. 2018.

KatZ, Michael; ShelansKi, Howard. 'Schumpeterian' Competition and Antitrust Policy in High-Tech Markets. Competition, [S.1.], v. 14, 2006, pp. 1-20.

KoCsIS, Viktória; WedA, Jarst. The innovation-enhancing effects of network neutrality, 2013. Disponível em: http://bit.ly/2EKRyhI. Acesso em: 9 jan. 2018.

LEMos, Ronaldo. O Marco Civil como símbolo do desejo por inovação no Brasil. In: LEITE, George Salomão (Coord.). Marco Civil da Internet. São Paulo: Atlas, 2014, pp. 3-11.

LESSIG, Lawrence. Code - version 2.0. Nova Iorque: Basic Books, 2006a. 
. Prepared Statement of Lawrence Lessig, C. Wendell and Edith M. Carlsmith Professor of Law, Stanford Law School, 2006b. Disponível em: http://bit.ly/2cqieKm. Acesso em: 9 jan. 2018.

LI, Zhu. Legal Boundaries of Competition in the Era of the Internet: Challenges and Judicial Responses. In: Aitor Ortiz (Ed.). Internet, competition and regulation of online platforms. Boston: CPI, 2016, pp. 143-154. Disponível em: http://bit.ly/2pIx9p7. Acesso em: 9 jan. 2018.

MANIADAKI, Katerina. EU Competiton Law, Regulation and the Internet - the case of Net Neutrality. Alphen aan den Rijn: Wolters Kluwer, 2015.

Mattos, Cesar et al. Nota Técnica: economia da neutralidade da rede. Revista do IBRAC Direito da Concorrência, Consumo e Comércio Exterior, São Paulo, v. 24, 2013, pp. 233274.

MARSDEN, Christopher T. Network neutrality: from policy to law to regulation. Manchester: Manchester University Press, 2017. Disponível em: http://bit.ly/2Bur4OU. Acesso em: 9 jan. 2018.

McCabe, David. FCC flooded with net neutrality complaints. The Hill, 30 jul. 2015. Disponível em: http://bit.ly/1kKNuTW. Acesso em: 9 jan. 2018.

Ministério da Ciência, TeCnologia, Inovações e Comunicações. Consulta Pública Estratégia Brasileira para a Transformação Digital, 2017. Disponível em: http://bit.ly/2u1NhET. Acesso em: 9 jan. 2018.

. Consulta Pública - Decreto de políticas de telecomunicações. Disponível em: http://bit.ly/2gPCm8Q. Acesso em: 9 jan. 2018.

. Ofício $\mathrm{n}^{\mathrm{o}}$ 40037/2016/SEI-MCTIC, 14 out. 2016. Disponível em: http://bit.ly/2CCdg6b. Acesso em: 9 jan. 2018.

MinistéRIO DAS COMUNICAÇõES. Norma 004/1995. Disponível em: http://bit.ly/1JqlL1D. Acesso em: 9 jan. 2018.

MPF. Denúncia Ofício $\mathrm{n}^{\circ}$ 144/2016 (MPF/CADE), 3 jun. 2016. Disponível em: http://bit.ly/2D5kr81. Acesso em: 9 jan. 2018. 
NAZAReno, Claudio. Texto de referência acerca do Marco Civil da Internet para o "Fique por dentro", 2014. Disponível em: http://bit.ly/2ACvyCv. Acesso em: 9 jan. 2018.

NiC.BR, Como funciona a Internet? Parte 1: o protocolo IP, 2014. Disponível em: http://bit.ly/2ansYZG. Acesso em: 9 jan. 2018.

Novais E SILVA, Leandro; LeURQuin, Pablo; Belfort, André. Os acordos de zero rating e seus impactos concorrenciais: os limites da regulação da neutralidade da rede. Revista de Defesa da Concorrência, Brasília, v. 4, 2016, pp. 21-56.

NUECHTERLEIN, Jonathan. Antitrust Oversight of an Antitrust Dispute - An Institutional Perspective on the Net Neutrality Debate. Journal on Telecommunications \& High Technology Law, Boulder, v. 7, 2009, pp. 19-66.

O'CONNER, Daniel. Understanding online platform competition: common misunderstandings. In: Aitor Ortiz (Ed.). Internet, competition and regulation of online platforms. Boston: CPI, 2016, pp. 9-29. Disponível em: http://bit.ly/2pIx9p7. Acesso em: 9 jan. 2018.

OCTAVIANI, Alessandro. Controle de condutas no direito econômico concorrencial brasileiro". In: Coelho, Fabio Ulhoa (Coord.). Tratado de Direito Comercial, v. 6, 2015, pp. 389-403.

OECD. Guia de Avaliação da Concorrência - Princípios, v. 1, 2007. Disponível em: http://bit.ly/2a9AWmU. Acesso em: 9 jan. 2018.

ONU, Comissão de Direitos Humanos. Resolução sobre a promoção, proteção e gozo dos direitos humanos na Internet (A/HRC/32/L.20), 2016. Disponível em: http://bit.ly/29jpSS4. Acesso em: 9 jan. 2018.

Opam, Kwame. After Comcast, Netflix signs traffic deal with Verizon - The streaming service cuts deals while net neutrality hangs in the balance. The Verge, 28 abr. 2014. Disponível em: http://bit.ly/1pHTEEK. Acesso em: 9 jan. 2018.

PeHA, Jon. The benefits and risks of mandating network neutrality, and the quest for a balanced policy, 2006. Disponível em: http://bit.ly/2a2KUET. Acesso em: 9 jan. 2018. 
Pereira Neto, Caio Mário da Silva; Casagrande, Paulo Leonardo. Direito Concorrencial doutrina, jurisprudência e legislação. São Paulo: Saraiva, 2016.

PFEIFFER, Roberto Augusto Castellanos. Defesa da concorrência e bem-estar do consumidor. São Paulo: Revista dos Tribunais, 2015.

PINHEIRO, Guilherme Pereira. O princípio da neutralidade de rede na Internet: uma análise regulatória e concorrencial. Prismas: Dir., Pol. Publ. e Mundial., Brasília, v. 5, n. 2, p. 235$254,2008$.

Poder Judiciário do Estado do Rio de Janeiro, Comarca de Duque de Caxias, $2^{\mathrm{a}}$ Vara Criminal, Juíza Daniela Barbosa Assumpção de Souza, IP 062-00164/2016, j. 19.07.2016. Disponível em: http://bit.ly/2aoyP0f. Acesso em: 9 jan. 2018.

Post, David. What Larry Doesn't Get: Code, Law, and Liberty in Cyberspace. Stanford Law Review, Stanford, v. 52, pp. 1439-1459, 2000. Disponível em: http://bit.ly/2coIgg6. Acesso em: 9 jan. 2018.

PriceWATERCOOPERs. Global Top 100 Companies by market capitalisation, 2017. Disponível em: https://pwc.to/2sS5M9z) . Acesso em: 9 jan. 2018.

PuZZANGHERA, Jim. Net neutrality rules formally published; first legal challenge filed. Los Angeles Times, 13 abr. 2015. Disponível em: http://lat.ms/1F2m6t4. Acesso em: 9 jan. 2018.

RAMOS JUNIOR, Durval. Estudo Mostra que NET é a principal inimiga dos torrents no Brasil. Tecmundo, 9 ago. 2012. Disponível em: http://bit.ly/29C7CSq. Acesso em: 9 jan. 2018.

RAMOS, Pedro Henrique Soares. Arquitetura da Rede e Regulação: a neutralidade da rede no Brasil. 2015a. 218 f. Dissertação (Mestrado em Direito) - Direito GV, Fundação Getúlio Vargas, São Paulo, 2015a.

O Marco Civil e a importância da neutralidade da rede: evidências empíricas. In: LuCCA, Newton de et al. Direito e Internet III - Tomo II. São Paulo: Quartier Latin, 2015b, pp. 137-154.

Reale, Miguel. Abuso do poder econômico e garantias individuais. In: FranceschinI, José Inácio Gonzaga; FrancessChinI, José Luiz Vicente de Azevedo. Poder econômico: exercício e abuso. São Paulo: Revista dos Tribunais, 1985, pp. 520-525. 
SAlOMÃo Filho, Calixto. Direito Concorrencial - as condutas. São Paulo: Malheiros, 2007.

. Regulação da atividade econômica (princípios e fundamentos jurídicos), $2^{\mathrm{a}}$ ed. São Paulo: Malheiros, 2008.

Direito Concorrencial. São Paulo: Malheiros, 2013.

Direito industrial, direito concorrencial e interesse público. Revista CEJ, Brasília, v. 35, 2006, pp. 12-19.

Saltzer, J.H.; ReED, D.P.; Clark, D.D. End-to-end arguments in system design. Disponível em: http://bit.ly/1pCN8kK. Acesso em: 9 jan. 2018.

Active Networking and End-To-End Arguments. Disponível em: http://bit.ly/2ljcFzE. Acesso em: 9 jan. 2018.

Santos Pinheiro, Juliana. Neutralidade de redes, instituições e desenvolvimento. $227 \mathrm{f}$. Tese (Doutorado em Políticas Públicas, Estratégias e Desenvolvimento) - Instituto de Economia, Universidade Federal do Rio de Janeiro, Rio de Janeiro, 2012.

SASHKIN, Davina. Failure of Imagination: why inaction on net neutrality will result in a de facto regime promoting discrimination and consumer harm. CommLaw Conspectus: Journal of Communications Law and Technology Policy, Washington D.C., v. 15, 2006, pp. 261309.

SCHEWICK, Barbara van. Internet architecture and innovation. Cambridge: MIT Press, 2010 .

; Frischmann, Brett. Network Neutrality and the Economics of an Information Superhighway - A Reply to Professor Yoo. Jurimetrics, Phoenix, v. 47, pp. 383-428, 2007. Disponível em: http://bit.ly/2cr1267. Acesso em: 9 jan. 2018.

Towards an economic framework for net neutrality regulation. Journal on Telecommunications and High Technology Law, Boulder, v. 5, 2007, pp. 329-391. 
SChumpeter, Joseph. Capitalism, Socialism and Democracy, $5^{\text {a }}$ ed. Routledge: Londres e Nova Iorque, 1984.

SEAE. Parecer analítico sobre regras regulatórias n. 03 COGCM/SEAE/MF - Contribuição à consulta pública sobre Minuta de Anteprojeto de Lei que estabelece o Marco Civil da Internet no Brasil, 2010. Disponível em: http://bit.ly/2aigujb. Acesso em: 9 jan. 2018.

Senado Federal, Comissão de Ciência e Tecnologia. Relatório de Avaliação do Programa Nacional de Banda Larga (PNBL) (Resolução no 44/2013), 2014. Disponível em: http://bit.ly/2a8xitE. Acesso em: 9 jan. 2018.

Shoemaker, Pamela J.; Vos, Tim P. Teoria do gatekeeping: seleção e construção da notícia, Trad. Vivian Nickel. Porto Alegre: Penso, 2011.

SIDAK, J. Gregory. The fallacy of "equal treatment" in Brazil's bill of rights for internet users. Revista Direito GV, São Paulo, v. 8, 2012, pp. 651-676.

A consumer-welfare approach to network neutrality regulation of the Internet. Journal of Competition Law and Economics, Oxford, v. 2(3), 2006, pp. 349-474.

SilveIRA, Newton; Godoy, Walter. Propriedade intelectual e liberdade. Revista de Direito Mercantil, Industrial, Econômico e Financeiro, São Paulo, v. 142, 2006, pp. 7-24.

Simão FilHo, Adalberto. Revisitando a nova empresarialidade a partir do Marco Civil em Contexto de Internet das Coisas. In: LuCCA, Newton de et al. Direito e Internet III - Tomo II. São Paulo: Quartier Latin, 2015, pp. 27-47.

Singel, Ryan. Vint Cerf: We knew what we were unleashing on the world. Wired, 23 abr. 2012. Disponível em: http://bit.ly/2ybk4bp. Acesso em: 9 jan. 2018.

SouZA, Affonso Pereira de. "As cinco faces da proteção à liberdade de expressão no Marco Civil da Internet". In: LuCCA, Newton de et al. Direito e Internet III - Tomo II. São Paulo: Quartier Latin, 2015, pp. 377-408.

; Moniz, Pedro de Paranaguá; VIEIRA Junior, Sérgio Branco. Neutralidade da rede, filtragem de conteúdo e interesse público: reflexões sobre o bloqueio do site YouTube no Brasil. Revista de Direito Administrativo, Belo Horizonte: Atlas, v. 246, 2007, pp. 71-73. 
SteIBEL, Fabro. O portal da consulta pública do Marco Civil da Internet. In: Lemos, Ronaldo; LEITE, George Salomão (Coord.). Marco Civil da Internet. São Paulo: Atlas, 2014, pp. 1828.

STF. Decisão Monocrática, MC na ADPF 403/SE, Min. Ricardo Lewandowski, j. 19.07.2016. Disponível em: http://bit.ly/2a4PO5u. Acesso em: 9 jan. 2018.

, Pleno. ADIn 319-4/DF, Rel. Min. Moreira Alves, j. 03.03.1993. Disponível em: http://bit.ly/2ieMlVQ. Acesso em: 9 jan. 2018.

Telesíntese, Neutralidade: presidente da Anatel lembra que no Brasil vale o Marco Civil da Internet, 15 dez. 2017. Disponível em: http://bit.ly/2Cxrqc0. Acesso em: 9 jan. 2018.

TIROLE, Jean; Rochet, Jean-Charle. Platform competition in two-sided markets. Journal of the European Economic Association, Cambridge MA, v. 1(4), 2003, pp. 990-1029.

Tomasevicius Filho, Eduardo. O Marco Civil e as Liberdade de Mercado. In: LuCCA, Newton de et al. Direito e Internet III - Tomo II. São Paulo: Quartier Latin, 2015, pp. 4964.

Toor, Amar. Europe's net neutrality guidelines seen as a victory for the open web. The Verge, 30 ago. 2016. Disponível em: http://bit.ly/2c88eSd. Acesso em: 9 jan. 2018.

UNIÃo EUROPEIA, Conselho da Europa. Regulamento europeu sobre neutralidade da rede, 2015. Disponível em: http://bit.ly/1Gv2FKQ. Acesso em: 9 jan. 2018.

WeISER, Mark; Brown, John Seely. The coming age of calm technology, 1996. Disponível em: http://bit.ly/1mfUsLh. Acesso em: 9 jan. 2018.

Weisman, Dennis; Kulick, Robert. Price discrimination, two-sided markets, and net neutrality regulation. Tulane Journal of Technology and Intellectual Property, Nova Orleans, v. 13, 2010, pp. 81-102.

Werbach, Kevin, A Layered Model for Internet Policy. Journal on Telecommunications and High Technology Law, Boulder, v.1, pp. 37-68, 2002. 
WeSTIN, Roberta. Neutralidade de rede: quem ganha e quem perde? In: ARTeSe, Gustavo. Marco Civil da Internet - análise jurídica sob uma perspectiva empresarial. São Paulo: Quartier Latin, 2015, pp. 135-157.

WizIACK, Julio. Teles preparam investida contra lei que proíbe cobrar mais na internet. Folha de São Paulo, 2 dez. 2017. Disponível em: http://bit.ly/2AQ1JTD. Acesso em: 9 jan. 2018.

et al. Oi pede recuperação judicial de $\mathrm{R} \$ 65$ bilhões, a maior da história do Brasil. Folha de São Paulo, 20 jun. 2016. Disponível em: http://bit.ly/2a8JE6w. Acesso em: 9 jan. 2018.

WoHLers, Marcio; ThOMAZINE, Jaqueline; SuAssuna, Priscila. Concorrência e inovação no mercado de produtos e serviços digitais: o debate sobre a neutralidade de redes em telecomunicações, 2010. Disponível em: http://bit.ly/21OLCf7 . Acesso em: 9 jan. 2018.

Wu, Tim. The Master Switch: the rise and fall of information empires. Nova Iorque: Vintage Books, 2010.

. Network Neutrality, Broadband Discrimination. Journal of Telecommunications and High Technology Law, Boulder, v. 2, 2003, pp. 141-178.

Network Neutrality: Competition, Innovation, and Nondiscriminatory Access, 2006. Disponível em: http://bit.ly/2zgmST7. Acesso em: 9 jan. 2018.

Yoo, Christopher. Would Mandating Broadband Network Neutrality Help or Hurt Competition? A Comment on the End-to-End Debate. Journal of Telecommunications and High Technology Law, Boulder, v. 3, 2004, pp. 23-68. Disponível em: http://bit.ly/2czKU3V. Acesso em: 9 jan. 2018.

. Beyond Network Neutrality. Harvard Journal of Law \& Technology, Cambridge, v. 19, 2005, pp. 1-77. Disponível em: http://bit.ly/2c6qdf7. Acesso em: 9 jan. 2018. 